\title{
Understanding and reconstructing the Asian climate of the last 2000 years
}

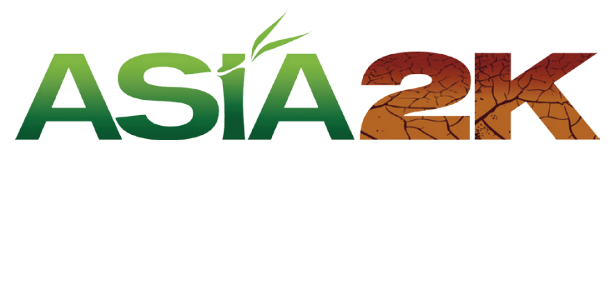

\author{
Quansheng Ge1, Z. Hao', X. Shao', H. Borgaonkar², J. Luterbacher³ , T. Nakatsuka4, M. Sano ${ }^{4}$, O. Solomina and L. Zhou $^{6}$ \\ $3^{\text {rd }}$ Asia2k workshop, Beijing, China, 26-27 May 2014
}

This workshop constituted the starting point for Phase II of Asia2k and was attended by 40 participants from 11 countries. They reviewed a variety of proxy records from Asia, coordinated the setup of a database following the selection criteria of the $2 \mathrm{k}$ Network, and agreed on a timeline towards the production and publication of regional spatial reconstructions of temperature and precipitation covering the past 2000 years.

Presentations addressed reconstruction methodologies, paleoclimate modeling, and paleoclimatic records from Asia based on historical documents and natural proxies. New, yet unpublished studies were presented. These included an extreme events record from historical documents, a tree-ring based temperature and drought reconstructions covering the past 2000 years from the Tien-Shan and Altay Mountains, a 2000-year long varved sediment record from northeastern China, a relative humidity variation record for the southwestern Gobi Desert reconstructed from oxygen isotopes in Qinghai spruce, and a two millennia long tree-ring cellulose oxygen isotope chronology from Japan. In addition, 500 year long tree-ring based precipitation records from the Nepal Himalayas, Northwestern Thailand and Pakistan were discussed. All these new proxy records are important contributions to the Asia2k database, especially since the Phase I database only included a limited number of records, all of which are tree ring series (Fig. 1).

To focus the discussions on the upcoming key tasks and plan the next specific steps of the Asia2k project, the participants broke out into three groups. Two groups focused on inventories of paleoclimatic records at high and low-resolution, respectively. Their aim was to develop strategies for compiling published (or soon to be published) proxy records of (sub-)annual to multi-decadal-resolution and their associated metadata from different paleoclimate archives. A third group focused on statistical reconstruction methods and ways forward to arrive at regional-scale spatially explicit reconstructions of temperature and precipitation variations.

In both data-related breakout groups participants agreed to contribute their data when published in papers, encouraged their colleagues to also contribute their datasets, and planned to extract other data from the existing literature. A coordinated publication, such as a special issue, was encouraged to support swift publication of yet unpublished data. The data submission process via the template downloadable from the $2 k$ website was found suitable. When reviewing the submission criteria, the high-resolution group expressed concern that a required minimum record length of 500 years would exclude too many good records. Accordingly, the 2k Network has in the meantime relaxed the criteria for annually-resolved records to a minimum length of 300 years. The low-resolution group on the other hand expressed concern that one date per 500 years would be a too strict criterion. In response, a more flexible definition for required chronological accuracy has been developed. The high-resolution group identified multi-decadal

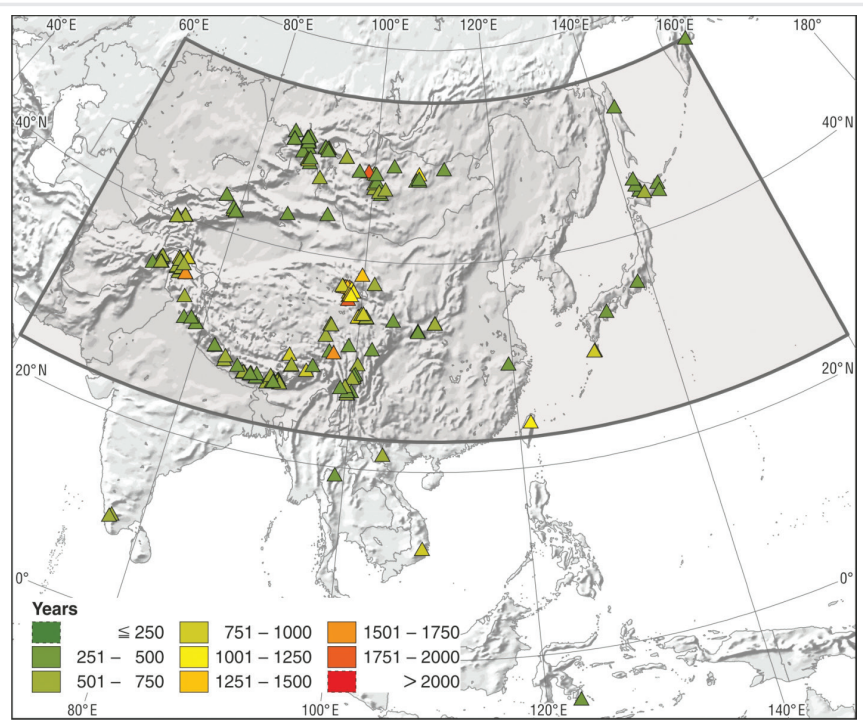

Figure 1: Phase I database of the Asia 2k group as published in the PAGES 2k Consortium paper (2013). A major task for the group is to add many of the available non-tree ring records to the database.

scale variability and extreme climatic events as the main scientific topics. In the low-resolution group the scientific topics of interest included multi-decadal to multi-centennial climatic changes such as of the meridional temperature gradient and of the treeline position.

The reconstruction group agreed to try different reconstruction methods, including downscaling from global reconstructions, and to compare the output with each other. The aim is to start with temperatures first, but not only for annual averages but also for seasonal temperatures. Experts agreed that a full spatial reconstruction of precipitation changes is unlikely to be achieved. Following the suggestion by the $2 \mathrm{k}$ Consortium (PAGES $2 \mathrm{k}$ Consortium 2014), the generation of independent reconstructions at high and low resolution will be considered, and Asia sub-divided into climatic regions if a full scale spatial reconstruction is not possible due to the lack of sufficient proxy records. Monsoon variability was identified as the scientific question of prime interest that could be addressed with the reconstructions.

The participants also discussed the new group and leadership structure. A steering committee of nine task leaders (the authors of this article) chaired by Quansheng Ge was established. Committee members include representatives of the four biggest countries, data management, and reconstruction methods.

The next step of the Asia $2 \mathrm{k}$ group is to update the temperature dataset by the end of the year 2014 and to apply a range of reconstruction methods to them. In parallel, the group will start compiling hydroclimatic proxy records. The fourth Asia $2 k$ workshop will be held in Japan in March 2015 with the goal being to compare and review the first-order reconstructions and finalize the data compilation.

\section{AFFILIATIONS}

IInstitute of Geographic Sciences and Natural Resources Research, Chinese Academy of Sciences, China; Indian Institute of Tropical Meteorology, Pune, India ${ }^{3}$ Department of Geography, Justus-Liebig-University Giessen, Germany

${ }^{4}$ Research Institute of Humanity and Nature, Kyoto, Japan ${ }^{5}$ Institute of Geography, Russian Academy of Sciences, Moscow, Russia

${ }^{6}$ Department of Geography, Peking University, Beijing, China

CONTACT

Zhixin Hao: haozx@igsnrr.ac.cn

REFERENCES

PAGES 2k Consortium (2013) Nat Geo 6: 339-346

PAGES 2k Consortium (2014) Eos 95: 361-368 